\title{
ON THE PILLAI-TIJDEMAN DIOPHANTINE EQUATION INVOLVING TERMS OF LUCAS SEQUENCES*
}

\author{
MAHADI DDAMULIRA, FLORIAN LUCA, AND ROBERT TICHY
}

\begin{abstract}
Let $r \geq 1$ be an integer and $\mathbf{U}:=\left\{U_{n}\right\}_{n \geq 0}$ be the Lucas sequence given by $U_{0}=0, U_{1}=$ 1 , and $U_{n+2}=r U_{n+1}+U_{n}$ for $n \geq 0$. In this paper, we explain how to find all the solutions of the Diophantine equation, $A U_{n}+B U_{m}=C U_{n_{1}}+D U_{m_{1}}$, in integers $r \geq 1,0 \leq m<n, 0 \leq m_{1}<n_{1}$, $A U_{n} \neq C U_{n_{1}}$, where $A, B, C, D$ are given integers with $A \neq 0, B \neq 0, m, n, m_{1}, n_{1}$ are nonnegative integer unknowns and $r$ is also unknown.
\end{abstract}

\section{INTRODUCTION}

Let $r \geq 1$ be an integer and $\mathbf{U}:=\left(U_{n}\right)_{n \geq 0}$ be the Lucas sequence given by $U_{0}=0, U_{1}=1$, and

$$
U_{n+2}=r U_{n+1}+U_{n}
$$

for all $n \geq 0$. When $r=1$, $\mathbf{U}$ coincides with the Fibonacci sequence while when $r=2$, $\mathbf{U}$ coincides with the Pell sequence.

Let

$$
(\alpha, \beta):=\left(\frac{r+\sqrt{r^{2}+4}}{2}, \frac{r-\sqrt{r^{2}+4}}{2}\right),
$$

be the roots of the characteristic equation $X^{2}-r X-1=0$ of the Lucas sequence $\mathbf{U}=\left(U_{n}\right)_{n \geq 0}$. It is easy to see that $\beta=-\alpha^{-1}$. The Binet formula for the general term of $\mathbf{U}$ is given by

$$
U_{n}:=\frac{\alpha^{n}-\beta^{n}}{\alpha-\beta} \text { for all } n \geq 0 .
$$

The divisibility property

$$
\operatorname{gcd}\left(U_{n}, U_{m}\right)=U_{\operatorname{gcd}(n, m)} \quad \text { for positive integers } n, m
$$

is well-known. It is heavily used in solving Diophantine equations involving members of Lucas sequences and it is an important ingredient in the proof of the Primitive Divisor Theorem for Lucas sequences (see [1] for such properties. In particular, the above property(3) appears as Proposition 2.1 (iii) in [1]). Furthermore, one can prove by induction that the inequality

$$
\alpha^{n-2} \leq U_{n} \leq \alpha^{n-1}
$$

holds for all positive integers $n$.

Shorey and Tijdeman [2] gave lower bounds for the absolute value and the greatest prime factor of the expression $A x^{m}+B y^{m}$ where $A, B, x, y, m \geq 0$ are integers. As an application, they proved, under suitable conditions, that the equation $A x^{m}+B y^{m}=C x^{n}+D y^{n}$ implies that $\max \{n, m\}$ is bounded by a computable constant depending only on $A, B, C, D$. More precisely, they proved the following result.

2010 Mathematics Subject Classification. 11B39, 11D61.

Key words and phrases. Lucas sequences, Diophantine equations.

* This note is dedicated to Robert Tijdeman on the occasion of his 80th birthday. 
Theorem 1. Let $A \neq 0, B \neq 0, C$, and $D$ be integers. Suppose that $x, y, m, n$ with $|x| \neq|y|$ and $0 \leq n<m$ are integers. There exists a computable constant $E$ depending only on $A, B, C, D$ such that the Diophantine equation

$$
A x^{m}+B y^{m}=C x^{n}+D y^{n}
$$

with

$$
A x^{m} \neq C x^{n}
$$

implies that $m \leq E$.

In this paper, we study a variation of the above result with the terms of the Lucas sequence $\mathbf{U}:=\left(U_{n}\right)_{n \geq 0}$. That is, we study the Diophantine equation

$$
A U_{n}+B U_{m}=C U_{n_{1}}+D U_{m_{1}} \quad \text { with } \quad n>m \geq 0 \quad \text { and } \quad n_{1}>m_{1} \geq 0, \quad A U_{n} \neq C U_{n_{1}} .
$$

Our first result is the following.

Theorem 2. Assume that $A, B, C, D$ are given integers, $A B \neq 0$ and $E q$. (7) holds. Then $r<14 X$, where $X:=\max \{|A|,|B|,|C|,|D|\}$.

Proof. Assume first that $C=D=0$. Then we take $m_{1}=0, n_{1}=1$. Then $A U_{n}=-B U_{m}$. If $m=0$, then $n=0$ which is not allowed. Thus, $m \neq 0$, so $U_{n} / U_{d}$ divides $B$, where $d:=\operatorname{gcd}(n, m)$. Write $n=: k d$, where $k \geq 2$. If $d=1$, then $U_{n} / U_{d}=U_{k} / U_{1}=U_{k} \geq U_{2}=r$, so $r \leq X$. If $d \geq 2$, then

$$
\frac{U_{n}}{U_{d}}=\frac{\alpha^{k d}-\beta^{k d}}{\alpha^{d}-\beta^{d}}
$$

We show that this last expression is $>\alpha$. This is equivalent to

$$
\alpha^{k d}>\alpha^{d+1}-\alpha \beta^{d}+\beta^{k d} \text {. }
$$

Since $d \geq 2,\left|\alpha \beta^{d}\right|=|\beta|^{d-1}<1$. Thus, it suffices that

$$
\alpha^{2 d}-\alpha^{d+1}>2 \text {. }
$$

The left-hand side is $\alpha^{d+1}\left(\alpha^{d-1}-1\right) \geq \alpha^{d+1}(\alpha-1)$. The smallest possible $\alpha$ is $\phi:=(1+\sqrt{5}) / 2$ (for $r=1$ ) and $\phi^{d+1}(\phi-1) \geq \phi^{3}(\phi-1)=\phi^{2}>2$. Thus, indeed $\alpha<U_{k d} / U_{d} \leq X$, which gives $r=\alpha+\beta<\alpha<X$. Further, $U_{n} \geq \alpha^{n-2}$ and $U_{d} \leq \alpha^{d-1}$ (by (4)), so

$$
\frac{U_{n}}{U_{d}} \geq \alpha^{n-d-3} \geq \alpha^{n-n / 2-3} \geq \alpha^{n / 2-3} .
$$

In the above we used that $d<n$ is a proper divisor of $n$, so $d \leq n / 2$. Since $U_{n} / U_{d}$ divides $B$, we get that $\alpha^{n / 2-3} \leq|B| \leq X$. Since $\alpha \geq \phi$, we get

$$
0<m<n \leq 6+2 \frac{\log X}{\log \phi} .
$$

This is when $C=D=0$.

So, we may assume that not both $C, D$ are 0 . If one of $C, D$ is nonzero and the other is zero, we assume that $C \neq 0$ and $n_{1} \neq 0$. Thus, if either $D=0$ or $m_{1}=0$, then the right-hand side is $C U_{n_{1}}$, otherwise it is $C U_{n_{1}}+D U_{m_{1}}$ with $D \neq 0$ and $n_{1}>m_{1}>0$. If $n=n_{1}$, then

$$
(A-C) U_{n}+B U_{m}=D U_{m_{1}} .
$$

The case $A-C=0$ is not allowed since then $A U_{n}=A U_{n_{1}}$. Thus, $A-C \neq 0$ and also $D \neq 0$. We also assume that $m \neq 0$ since if $m=0$, we are in the preceeding case. So, if $n=n_{1}$, then we replace $(A, B, C, D)$ by $(A-C, B, D, 0)$. The only effect is that $X$ is replaced by $2 X$. Thus, we 
may assume that $n \neq n_{1}$, and switching $A$ with $C$, if needed, we may assume that $n=\max \left\{n, n_{1}\right\}$, therefore $n>n_{1}$. We relabel our indices $\left(n, m, n_{1}, m_{1}\right)$ as $\left(n_{1}, n_{2}, n_{3}, n_{4}\right)$ where $n_{1}>n_{2} \geq n_{3} \geq n_{4}$, and the coefficients $A, B, C, D$ as $A_{1}, A_{2}, A_{3}, A_{4}$ and change signs to at most a couple of them so that our equation is now

$$
A_{1} U_{n_{1}}+A_{2} U_{n_{2}}+A_{3} U_{n_{3}}+A_{4} U_{n_{4}}=0 .
$$

Furthermore, $A_{1}, A_{2}, A_{3}$ are all nonzero but $A_{4}$ (or $n_{4}$ ) might be 0 . This leads to

$$
\left|A_{1}\right| \alpha^{n_{1}}=\left|-A_{2}\left(\alpha^{n_{2}}-\beta^{n_{2}}\right)-A_{3}\left(\alpha^{n_{3}}-\beta^{n_{3}}\right)-A_{4}\left(\alpha^{n_{4}}-\beta^{n_{4}}\right)+A_{1} \beta^{n_{1}}\right|<7 X \alpha^{n_{2}},
$$

so

$$
\alpha^{n_{1}-n_{2}}<7 X
$$

Thus, since $n_{1}>n_{2}$, we get that $r<\alpha \leq \alpha^{n_{1}-n_{2}}<7 X$. Recalling that we might have to replace $X$ by $2 X$, we get the desired conclusion.

\section{Finding ALL SOLUTiONS}

So far, we know that $r$ is bounded. It is possible for small $r$ that the equation has infinitely many solutions. By the preceding analysis, we saw that this is not the case if $C=D=0$, since then $0<n<6+2 \log X / \log \phi$. So, we assume that not both $C$ and $D$ are zero. Using the substitution $(A, B, C, D) \mapsto(A-C, B, D, 0)$, and relabelling some of the variables, we may assume that $n_{1}>n_{1} \geq n_{3} \geq n_{4}$ and that equation (9) holds. Then estimate (10) holds, so

$$
n_{1}-n_{2}<\frac{\log (7 X)}{\log \phi}
$$

We return to (9) and rewrite it as

$$
\left|\alpha^{n_{2}}\left(A_{1} \alpha^{n_{1}-n_{2}}+A_{2}\right)-\left(\frac{A_{1}}{(-\alpha)^{n_{1}}}+\frac{A_{2}}{(-\alpha)^{n_{2}}}\right)\right|=\left|-A_{3}\left(\alpha^{n_{3}}-\beta^{n_{3}}\right)-A_{4}\left(\alpha^{n_{4}}-\beta^{n_{4}}\right)\right| .
$$

The right-hand side is $\leq 4 X \alpha^{n_{3}}$. In the left-hand side we have $n_{1}-n_{2}>0$, so $A_{1} \alpha^{n_{1}-n_{2}}+A_{2} \neq 0$. Thus,

$$
\left|A_{1} \alpha^{n_{1}-n_{2}}+A_{2}\right|\left|A_{1} \beta^{n_{1}-n_{2}}+A_{2}\right| \geq 1 .
$$

The second factor in the left above is $\leq 2 X$. Thus, $\left|A_{1} \alpha^{n_{1}-n_{2}}+A_{2}\right| \geq 1 / 2 X$. Further,

$$
\left|\frac{A_{1}}{(-\alpha)^{n_{1}}}+\frac{A_{2}}{(-\alpha)^{n_{2}}}\right| \leq \frac{2 X}{\alpha^{n_{2}}}
$$

Hence,

Assume first that

$$
\left|\alpha^{n_{2}}\left(A_{1} \alpha^{n_{1}-n_{2}}+A_{2}\right)-\left(\frac{A_{1}}{(-\alpha)^{n_{1}}}+\frac{A_{2}}{(-\alpha)^{n_{2}}}\right)\right| \geq \frac{\alpha^{n_{2}}}{2 X}-\frac{2 X}{\alpha^{n_{2}}} .
$$

$$
\frac{\alpha^{n_{2}}}{2 X}-\frac{2 X}{\alpha^{n_{2}}} \leq \frac{\alpha^{n_{2}}}{4 X}
$$

Then $\alpha^{2 n_{2}}<8 X^{2}$, so $\alpha^{n_{2}}<3 X$. Hence,

$$
n_{2}<\frac{\log (3 X)}{\log \phi}
$$

which together with 10 gives

$$
n_{4} \leq n_{3} \leq n_{2} \leq \frac{\log (3 X)}{\log \phi} \quad \text { and } \quad n_{1}<\frac{\log \left(21 X^{2}\right)}{\log \phi} .
$$


This was assuming (12) holds. Otherwise,

$$
\frac{\alpha^{n_{2}}}{4 X} \leq \frac{\alpha^{n_{2}}}{2 X}-\frac{2 X}{\alpha^{n_{2}}} \leq 4 X \alpha^{n_{3}}
$$

so

$$
\alpha^{n_{2}-n_{3}} \leq 16 X^{2}
$$

Hence, we get

$$
n_{2}-n_{3} \leq 2 \frac{\log (4 X)}{\log \phi}
$$

We rewrite equation (9) as

$$
\left|\alpha^{n_{3}}\left(A_{1} \alpha^{n_{1}-n_{3}}+A_{2} \alpha^{n_{2}-n_{3}}+A_{3}\right)-\left(\frac{A_{1}}{(-\alpha)^{n_{1}}}+\frac{A_{2}}{(-\alpha)^{n_{2}}}+\frac{A_{3}}{(-\alpha)^{n_{3}}}\right)\right|=\left|-A_{4}\left(\alpha^{n_{4}}-\beta^{n_{4}}\right)\right| .
$$

Assume first that

$$
A_{1} \alpha^{n_{1}-n_{3}}+A_{2} \alpha^{n_{2}-n_{3}}+A_{3}=0 .
$$

Let $i=n_{2}-n_{3}, j=n_{1}-n_{3}$. Then

$$
j=\left(n_{1}-n_{2}\right)+\left(n_{2}-n_{3}\right) \leq \frac{\log \left(112 X^{3}\right)}{\log \phi} \quad \text { and } \quad i \leq 2 \frac{\log (4 X)}{\log \phi}
$$

are bounded. Thus, one computes all polynomials $A_{1} X^{j}+A_{2} X^{i}+A_{3}$ and checks which of them has a root $\alpha$ which is a quadratic unit of norm -1 . For these Lucas sequences, it is the case that also $\beta$ is a root of the same polynomial so that the left-hand side of $(16)$ is zero for any $n_{3}$. This shows that also $n_{4}=0$. Thus, we have that

$$
\left(n_{1}, n_{2}, n_{3}, n_{4}\right)=\left(n_{3}+i, n_{3}+j, n_{3}, 0\right)
$$

is a parametric family of solutions. From now on we assume that the expression shown at (17) is nonzero. Then

$$
\left|A_{1} \alpha^{n_{1}-n_{3}}+A_{2} \alpha^{n_{2}-n_{3}}+A_{3}\right|\left|A_{1} \beta^{n_{1}-n_{2}}+A_{2} \beta^{n_{2}-n_{3}}+A_{3}\right| \geq 1 .
$$

The second factor in the left-hand side is $\leq 3 X$, therefore we conclude that

$$
A_{1} \alpha^{n_{1}-n_{3}}+A_{2} \alpha^{n_{2}-n_{3}}+A_{3} \mid \geq \frac{1}{3 X} \text {. }
$$

Further,

$$
\left|\frac{A_{1}}{(-\alpha)^{n_{1}}}+\frac{A_{2}}{(-\alpha)^{n_{2}}}+\frac{A_{3}}{(-\alpha)^{n_{3}}}\right| \leq \frac{3 X}{\alpha^{n_{3}}} .
$$

Hence, assuming (17) does not hold, the left-hand side of $(16)$ is at least as large as

$$
\frac{\alpha^{n_{3}}}{3 X}-\frac{3 X}{\alpha^{n_{3}}} .
$$

We distinguish two cases. If

$$
\frac{\alpha^{n_{3}}}{3 X}-\frac{3 X}{\alpha^{n_{3}}} \leq \frac{\alpha^{n_{3}}}{6 X},
$$

we then get $\alpha^{2 n_{3}}<18 X^{2}$, so $\alpha^{n_{3}} \leq 5 X$. Hence,

$$
n_{3} \leq \frac{\log (5 X)}{\log \phi} .
$$


Together with (10) and (15), we get

$$
\begin{aligned}
& n_{4} \leq n_{3} \leq \frac{\log (5 X)}{\log \phi}, \\
& n_{2} \leq\left(n_{2}-n_{3}\right)+n_{3} \leq \frac{\log \left(80 X^{3}\right)}{\log \phi}, \\
& n_{1} \leq\left(n_{1}-n_{2}\right)+n_{2} \leq \frac{\log \left(560 X^{4}\right)}{\log \phi} .
\end{aligned}
$$

Note that $(20)$ contains $(14)$. Finally assume that (18) does not hold. Then the left-hand side of $(16)$ is at least

$$
\frac{\alpha^{n_{3}}}{6 X}
$$

Comparing with the right-hand side of 16 we get

$$
\frac{\alpha^{n_{3}}}{6 X} \leq 2 X \alpha^{n_{4}} \leq 2 X \alpha^{n_{4}},
$$

so $\alpha^{n_{3}-n_{4}} \leq 12 X^{2}$. Thus,

$$
n_{3}-n_{4} \leq \frac{\log \left(12 X^{2}\right)}{\log \phi}
$$

Finally, we rewrite our equation as

$$
\alpha^{n_{4}}\left(A_{1} \alpha^{n_{1}-n_{4}}+A_{2} \alpha^{n_{2}-n_{4}}+A_{3} \alpha^{n_{3}-n_{4}}+A_{4}\right)=\beta^{n_{4}}\left(A_{1} \beta^{n_{1}-n_{4}}+A_{2} \beta^{n_{2}-n_{4}}+A_{3} \beta^{n_{3}-n_{4}}+A_{4}\right) .
$$

The exponents $i=n_{3}-n_{4}, j=n_{2}-n_{4}, k=n_{1}-n_{4}$ have only finitely many values. In fact,

$$
\begin{aligned}
& i \leq \frac{\log \left(12 X^{2}\right)}{\log \phi}, \\
& j=i+\left(n_{2}-n_{3}\right) \leq \frac{\left(\log \left(12 X^{2}\right)+\log \left(16 X^{2}\right)\right)}{\log \phi} \leq \frac{\log \left(200 X^{3}\right)}{\log \phi}, \\
& k=j+\left(n_{1}-n_{2}\right) \leq \frac{\left(\log \left(200 X^{3}\right)+\log (7 X)\right)}{\log \phi}=\frac{\log \left(1400 X^{4}\right)}{\log \phi} .
\end{aligned}
$$

So, we take all such polynomials $A X^{k}+A_{2} X^{j}+A_{3} X^{i}+A_{4}$ and search which ones of them have a root $\alpha$ which is a quadratic unit of norm -1 . For such, (22) holds for all $n_{4}$. Hence, we got the parametric family

$$
\left(n_{1}, n_{2}, n_{3}, n_{4}\right)=\left(n_{4}+k, n_{4}+j, n_{4}+i, n_{4}\right) .
$$

Assume next the the left-hand side of $(22)$ is nonzero. Then

$$
\left|A_{1} \alpha^{n_{1}-n_{4}}+A_{2} \alpha^{n_{2}-n_{4}}+A_{3} \alpha^{n_{3}-n_{4}}+A_{4}\right|\left|A_{1} \beta^{n_{1}-n_{4}}+A_{2} \beta^{n_{2}-n_{4}}+A_{3} \beta^{n_{3}-n_{4}}+A_{4}\right| \geq 1 .
$$

The second factor on the left-hand side above is $\leq 4 X$. Hence,

$$
\left|A_{1} \alpha^{n_{1}-n_{4}}+A_{2} \alpha^{n_{2}-n_{4}}+A_{3} \alpha^{n_{3}-n_{4}}+A_{4}\right| \geq \frac{1}{4 X} .
$$

Hence, in 22, we get

$$
\frac{\alpha^{n_{4}}}{4 X} \leq 4 X|\beta|^{n_{4}}=\frac{4 X}{\alpha^{n_{4}}},
$$

which gives

$$
n_{4} \leq \frac{\log (4 X)}{\log \phi}
$$


This together with (10), (15) and (21) gives

$$
\begin{aligned}
& n_{4} \leq \frac{\log (4 X)}{\log \phi}, \\
& n_{3} \leq\left(n_{3}-n_{4}\right)+n_{4} \leq \frac{\log \left(50 X^{3}\right)}{\log \phi}, \\
& n_{2} \leq\left(n_{2}-n_{3}\right)+n_{3} \leq \frac{\log \left(1000 X^{5}\right)}{\log \phi}, \\
& n_{1} \leq\left(n_{1}-n_{2}\right)+n_{2}<\frac{\log \left(10000 X^{6}\right)}{\log \phi} .
\end{aligned}
$$

Note that (24) contains (20) and (8). Recalling that we have to replace $X$ by $2 X$, we got the following theorem which is our second result.

Theorem 3. Let $\phi:=(1+\sqrt{5}) / 2$ be the smallest possible $\alpha$. Relabeling the variables $\left(n, m, n_{1}, m_{1}\right)$ to $\left(n_{1}, n_{2}, n_{3}, n_{4}\right)$, where $n_{1} \geq n_{2} \geq n_{3} \geq n_{4}$. If $n_{1}=n_{2}$, we rewrite the Diophantine equation (7) as

$$
(A-C) U_{n}+B U_{m}=D U_{m_{1}},
$$

and change $(A, B, C, D)$ to $(A-C, B, D, 0)$. Thus, $n_{1}>n_{2}$. Furthermore, we change the sign of some of the coefficients $(A, B, C, D)$ so that the Diophantine equation (7) becomes

$$
A_{1} U_{n_{1}}+A_{2} U_{n_{2}}+A_{3} U_{n_{3}}+A_{4} U_{n_{4}}=0 .
$$

Assume $r \leq 14 X$. Then, the solutions of the Diophantine equation (25) are of two types:

(i) Sporadic ones. These are finitely many and they satisfy:

$$
\begin{aligned}
& n_{4} \leq \frac{\log (8 X)}{\log \phi}, \quad n_{3} \leq \frac{\log \left(400 X^{3}\right)}{\log \phi}, \\
& n_{2} \leq \frac{\log \left(32000 X^{5}\right)}{\log \phi}, \quad n_{1} \leq \frac{\log \left(640000 X^{6}\right)}{\log \phi} .
\end{aligned}
$$

(ii) Parametric ones. These are of one of the two forms:

$$
\left(n_{1}, n_{2}, n_{3}, n_{4}\right)=\left(n_{3}+j, n_{3}+i, n_{3}, 0\right),
$$

where

$$
i \leq 2 \frac{\log (8 X)}{\log \phi} \quad \text { and } \quad j \leq \frac{\log \left(500 X^{3}\right)}{\log \phi},
$$

and $\alpha$ is a root of $A_{1} X^{i}+A_{2} X^{j}+A_{3}=0$, or of the form

$$
\left(n_{1}, n_{2}, n_{3}, n_{4}\right)=\left(n_{4}+k, n_{4}+j, n_{4}+i, n_{4}\right),
$$

where

$$
i \leq \frac{\log \left(50 X^{2}\right)}{\log \phi}, \quad j \leq \frac{\log \left(1600 X^{3}\right)}{\log \phi}, \quad k \leq \frac{\log \left(25000 X^{4}\right)}{\log \phi},
$$

and $\alpha$ is a root of

$$
A_{1} X^{k}+A_{2} X^{j}+A_{3} X^{i}+A_{4}=0
$$




\section{NUMERICAL EXAMPLES}

Just for fun, we took $A_{1}, A_{2}, A_{3}, A_{4} \in\{0, \pm 1\}$. Hence, $X=1$, therefore $r \leq 14$. Thus, Theorem 3 says that the sporadic solutions are of the form

$$
U_{n_{1}} \pm A_{2} U_{n_{2}} \pm A_{3} U_{n_{3}} \pm A_{4} U_{n_{4}}=0, \quad A_{2}, A_{3}, A_{4} \in\{0, \pm 1\}, n_{1}>n_{2} \geq n_{3} \geq n_{4} \geq 0 .
$$

Here, $n_{4} \leq 4, n_{3} \leq 12, n_{2} \leq 21$ and $n_{1}>n_{2}$. To search for them, we searched for $r \in[1,14]$, $n_{4} \in[0,4], n_{3} \in\left[n_{4}, 12\right], n_{2} \in\left[n_{3}, 21\right], \varepsilon_{4} \in\{0,1\}, \varepsilon_{3} \in\{0, \pm 1\}, \varepsilon_{2} \in\{0, \pm 1\}$ such that

$$
U_{n_{1}}=\left|\varepsilon_{2} U_{n_{2}}+\varepsilon_{3} U_{n_{3}}+\varepsilon_{4} U_{n_{4}}\right| \text { holds for some } n_{1}>n_{2} .
$$

A Mathematica code running for a few seconds found 207 solutions. Of them 194 correspond to the Fibonacci sequence $(r=1), 12$ correspond to the Pell sequence $(r=2)$ and only one of them namely $U_{1}+U_{1}+U_{1}=U_{2}$ corresponds to $r=3$. For parametric ones, Theorem 3 says that we need to find positive integers $i \leq 8, j \leq 15, k \leq 21$ such that $X^{k}+\varepsilon_{1} X^{j}+\varepsilon_{2} X^{i}+\varepsilon_{3}$ is a multiple of $X^{2}-r X-1$ for some $r \in[1,14]$, where $\varepsilon_{1} \in\{0, \pm 1\}, \varepsilon_{2} \in\{0, \pm 1\}, \varepsilon_{3} \in\{ \pm 1\}$. The only such instances found were $r=1$ for which only $X^{2}-X-1$ and $X^{4}-X^{3}-X-1$ were multiples of $X^{2}-r X-1=X^{2}-X-1$. These two instances lead to the parametric families

$$
F_{n+2}-F_{n+1}-F_{n}-F_{0}=0 \quad \text { and } \quad F_{n+4}-F_{n+3}-F_{n+1}-F_{n}=0,
$$

which hold for all $n \geq 0$. Enlarging $X$ (so, say allowing $A_{1}, A_{2}, A_{3}, A_{4}$ in $[-X, X], A_{1} \neq 0$ for a fixed integer $X \geq 2$ ) would of course detect more sporadic solutions and more parametric families involving the Pell sequence, etc. We leave pursuing such numerical investigations for the interested reader.

\section{Comments}

In this paper, we worked with the Lucas sequence $\left(U_{n}\right)_{n \geq 0}$ of characteristic equation $X^{2}-r X-1=$ 0 , where $r \geq 1$ is also a variable. Similar arguments can be used to deal with the equation (7) when the characteristic equation of $\left(U_{n}\right)_{n \geq 0}$ is $X^{2}-r X-s=0$, where $s$ is a fixed nonzero integer. The conclusion should be the same, namely that for given $A, B, C, D$, equation (7) implies that all its solutions come in two flavours; namely sporadic (maybe none) solutions whose indices $\max \left\{n, n_{1}\right\}$ are bounded by a computable function $f(X, s)$, depending on $X$ and $s$; and possibly additional parametric solutions namely of the form $\left(n, m, n_{1}, m_{1}\right)=(n, n-i, n-j, n-k)$, where $i, j, k$ are bounded by some computable function $g(X, s)$ depending on $X$ and $s$, and $n$ is a free parameter. Again, we leave pursuing such endeavours to the interested reader.

\section{ACKNOWLEDGEMENTS}

M. D. and R. T. were supported by the Austrian Science Fund (FWF) projects: F5510-N26 - Part of the special research program (SFB), "Quasi-Monte Carlo Methods: Theory and Applications" and W1230 - "Doctoral Program Discrete Mathematics". F. L. was supported by grant RTNUM19 from CoEMaSS, Wits, South Africa.

\section{REFERENCES}

[1] Yu. Bilu, G. Hanrot and P. M. Voutier, Existence of primitive divisors of Lucas and Lehmer numbers (with an appendix by M. Mignotte), J. Reine Angew. Math. 539 (2001), 75-122.

[2] T. N. Shorey and R. Tijdeman, Exponential Diophantine Equations, Cambridge Tracts in Mathematics (87), Cambridge University Press, 1986. 


\author{
MAHADi DDAMULIRA \\ Department of Mathematics, Makerere University, \\ P.O. Box 7062 Kampala, Uganda \\ Max Planck Institute for Software Systems, Saarland Informatics Campus, \\ Campus E1 5, D-66123 SaArbrüCKen, Germany \\ Email address: mahadi.ddamulira@mak.ac.ug; mddamulira@mpi-sws.org \\ FLORIAN LUCA \\ School of Mathematics, University of the WitwatersRand, \\ Johannesburg, South Africa \\ Research Group in Algebraic Structures and Applications, King Abdulaziz University, \\ JEDDAh, SAUdi Arabia \\ Max Planck Institute for Software Systems, SaArland Informatics Campus, \\ Campus E1 5, D-66123 Saarbrücken, Germany \\ Centro de Ciencias Matemáticas UnAm \\ Morelia, MeXico \\ Email address: Florian.Luca@wits.ac.za \\ ROBERT TICHY \\ Institute of Analysis and Number Theory, Graz University of Technology, \\ KopernikUsGasse 24/II, \\ A-8010 Graz, Austria \\ Email address: tichy@tugraz.at
}

